\title{
Synthesis and Characterization of some new $\beta$-Lactam Derivatives from Azo Sulphadiazine and its Biological Evaluation as Anticancer
}

\author{
RADHIYAH A. KHDUR and EZZAT H. ZIMAM \\ Department of Chemistry, Faculty of Education for Girls, the University of Kufa, Iraq. \\ Department of Chemistry, Faculty of Science, the University of Kufa, Iraq. \\ ${ }^{*}$ Corresponding author E-mail: radhiyah.aldujaili@uokufa.edu.iq \\ http://dx.doi.org/10.13005/ojc/340140
}

(Received: June 28, 2017; Accepted: October 03, 2017)

\section{ABSTRACT}

In this work new Azo compound was synthesized through a diazo-coupling reaction (S1) (4-amino-N-(pyrimidine-2-yl)-3-( pyrimidine-2-yldiazenyl)benzene sulfonamide). The diazotization process of 2-aminopyrimidin with Sulphadiazine as coupling component was described ,then Schiff bases[B1-B5] prepared from condensation of the free amino group in the azo compound with many aromatic aldehydes by using glacial Acetic acid as solvent. Cyclization of Shiff bases with Chloroacetyl chloride in the presence of tri ethyl amine give the corresponding $\beta$-lactam derivatives [L1-L5].All these compounds identified by( FTIR , $\left.{ }^{1} \mathrm{H}-\mathrm{NMR},{ }^{13} \mathrm{C}-\mathrm{NMR}\right)$ and follow by (TLC) and melting points of them.

Keywords: 2-amino pyrimidine, Sulphadiazine, Schiff base, $\beta$-Lactam.

\section{INTRODUCTION}

Sulfadiazine is a sulfonamide antibiotic and it is recognized as one of" the World Health Organization's List of Essential Medicines", it removes bacteria that cause infections by stopping the production of folic acid into the bacterial cell, and is Usually used to" treat urinary tract infections" (UTIs) and burns ${ }^{1,2}$. Schiff bases are Compounds containing an azo methine group $(-\mathrm{CH}=\mathrm{N})$ which formed by the condensation of an active carbonyl compound with primary amine, this reaction is recognized to be reversible and the removal of water to drive the reaction to completion is often fundamental to gain a good yield ${ }^{3,4,5}$. Schiff bases are the important compounds due to their wide range of industrial application and biological activities. they have been reported to posses the

This is an Open Access article licensed under a Creative Commons Attribution-NonCommercial-ShareAlike 4.0 International License (https://creativecommons.org/licenses/by-nc-sa/4.0/ ), which permits unrestricted NonCommercial use, distribution and reproduction in any medium, provided the original work is properly cited. 
pharmacological activities such as antibacterial, anti-malarial, anti-fungal, antioxidant, anti-cancer, anti-tubercular, anti-inflammatory, insecticidal and anticonvulsant also used as versatile component with organo-metallic reagents in nucleophilic addition and in cyclic addition reactions ${ }^{6.7,8}$. $\beta$-lactam (2-Azetidinone) are four-membered cyclic amide that which derived from 3-amino-propanoic acid ${ }^{9}$. It is named so because the $(\mathrm{N})$ atom is connected to the $\beta$-carbon atom close to the carbonyl ${ }^{10}$. The $\beta$-lactam ring is portion of the core structure of several antibiotic families, the major ones being the cephalosporins, penicillins, carbapenems, and monobactams, which are also called ( $\beta$-lactam antibiotics). Almost all of these antibiotics work by inhibition bacterial cell wall biosynthesis ${ }^{11}$. The first synthetic $\beta$-lactam ring was prepared by Hermann Staudinger in(1907) by reaction of the Schiff base ofbenzaldehyde and aniline with diphenylketene in a $[2+2]$ cycloaddition $^{12,13}$. A new research has suggested that $\beta$-lactams can subject ring-opening polymerization to form the amide bonds, to become "nylon-3 polymers". The basis of these polymers are similar to peptides, which offer them biofunctionality. nylon-3 polymers can either mimic host defense peptides or act as signals to stimulate 3T3 stem cell function ${ }^{14}$.

This research involve synthesis of some new $\beta$-lactam derivatives mixed with azo compound and study biological activity of the prepared compounds.

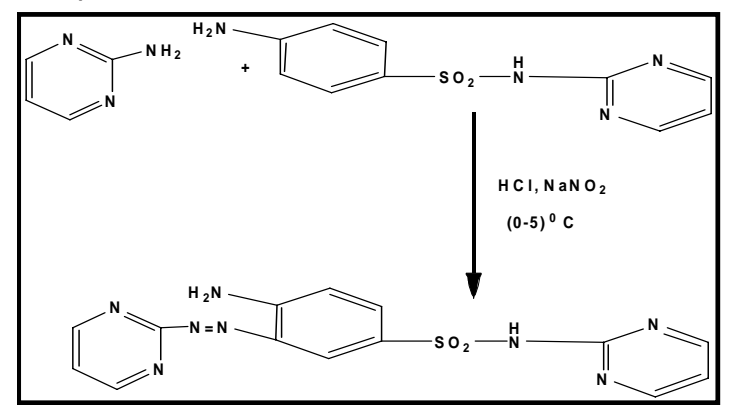

Scheme. 1: diazo-coupling reaction

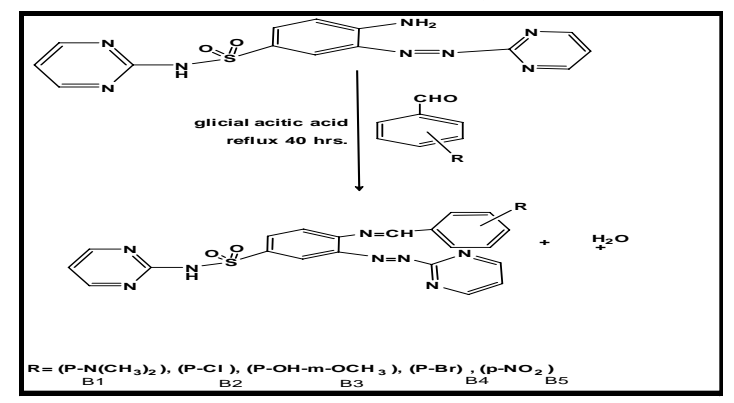

Scheme. 2: Preparation of Shiff Bases

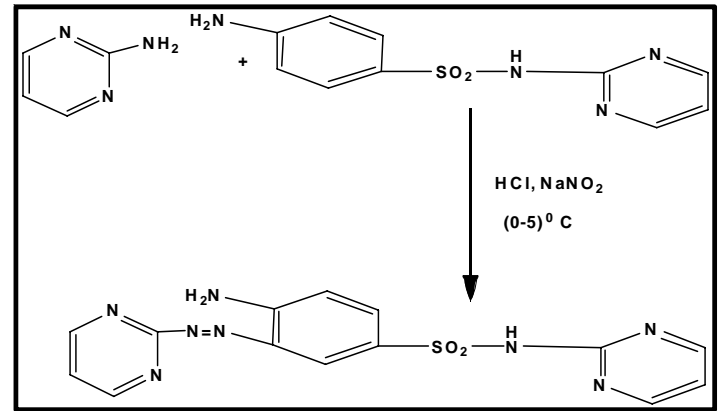

Scheme. 3: preparation of B-Lactam derivatives

\section{EXPERIMENTAL}

All chemicals were of highest purity and supplied by Merck and Fluka-company. Measurements melting points were recorded by using electro thermal 9300," melting point engineering LTD , U.K". Thin layer chromatography (T.L.C)was performed on silica gel for and spots were visualized by lodine vapors." FTIR" spectra, Fourier transform infrared shimadzu (8400), $\mathrm{H}^{1}$-NMR \& $\mathrm{C}^{13}$-NMR-spectra in (ppm) unit were operating in DMSO - $d 6$ as solvent using (BrukerUltra Shield $300 \mathrm{MHz}$ Switzerland)- (Iran).

Synthesis of 4-Amino-N-(Pyrimidine-2-yl)-3( Pyrimidine-2 yldiazenyl)benzene sulfonamide $(\mathrm{Azo})\left(\mathrm{S}_{1}\right)^{15}$ :

2-amino Pyrimidine (0.01 mole)( $0.95 \mathrm{~g})$ was dissolved in (4 ml) of concentrated hydrochloric acid and $(15 \mathrm{ml})$ of distilled water The mixture was cooled at $\left(0-5^{\circ} \mathrm{C}\right)$ in ice-water bath, Then a solution of sodium nitrite ( 0.01 mole) $(0.69 \mathrm{~g}$ ) was dissolved in $(10 \mathrm{ml})$ of distilled water then it will be cooled at $\left(0-5^{\circ} \mathrm{C}\right)$. This solution was added a drop wise to the mixture with stirring at the same temperature. The diazonum salt solution was added portion wise to solution of $(0.01 \mathrm{~mol}, 2.5 \mathrm{~g})$ sulfadiazine in distilled water with sodium hydroxide $(5 \mathrm{~g})$ dissolved in $(100 \mathrm{ml})$ distilled water. The basicity was nutralise by adding drops of $(\mathrm{HCl})$ until the $\mathrm{pH}$ become (7) and temperature was maintained at $\left(0-5^{\circ} \mathrm{C}\right)$. The mixture was stirred for $30 \mathrm{~min}$. and was left over night. The product was precipitated and filtered, washed well with distilled water and re-crystallized from absolute ethanol, yield ( $89.5 \%$ ) m.p. $\left(248-249{ }^{\circ} \mathrm{C}\right)$. 


\section{I.R spectra}

$\left(\mathrm{NH}_{2}\right)$ str. $(3427-3358) \mathrm{cm}^{-1}, \mathrm{Azo}(1440-$ 1492) $\mathrm{cm}^{-1},(\mathrm{C}=\mathrm{C})$ str.Aromatic $(1585) \mathrm{cm}^{-1},(\mathrm{~N}-\mathrm{H})$ str. Sulfone $(3257) \mathrm{cm}^{-1},(C=N)$ str. Pyrimidine (1666-1635) $\mathrm{cm}^{-1},(\mathrm{C}-\mathrm{H})$ str. Aromatic (3039) $\mathrm{cm}^{-1}, \mathrm{SO} 2(1325) \mathrm{cm}^{-1}$.

\section{${ }^{1}$ HNMR spectrum}

( $\delta \mathrm{ppm}),(\mathrm{DMSO}-\mathrm{d} 6 \mathrm{MHz}),\left(\mathrm{NH}_{2)}(6.015)\right.$ $((\mathrm{Ar}-\mathrm{H})$ (6.559-7.033), $(\mathrm{HC}=\mathrm{N})$ pyrimidine $(7.612-$ 8.498), (N-H) Sulfone (11.294), DMSO(2.5).

\section{${ }^{13} \mathrm{C}$-NMR-spectrum}

( $\delta \mathrm{ppm}),(\mathrm{DMSO}-d 6, \mathrm{MHz}), 110.56,112.59$ 115.99, 125.33, 130.28, 153.50, 157.69 , 158.47, 158. 71.

\section{General procedure for synthesis Schiff bases [B1-B5] ${ }^{16}$}

Azo Compound $\left(S_{1}\right)(0.01 \mathrm{~mol})$ was dissolved in hot glacial acetic acid about $(50 \mathrm{ml})$ then added to $(0.01 \mathrm{~mol})$ of different aromatic aldehydes were dissolved in $(5 \mathrm{ml})$ of glacial acetic acid. The reaction mixture was refluxed at $\left(100^{\circ} \mathrm{C}\right)$ with stirring for 40 hours. The progress of the reaction was followed by TLC. After the completion the mixture was poured onto ice crushed. The yielded solid was filtered off and wash with (2\%) Sodium bicarbonate solution and distilled water then recrystallized from absolute Ethanol.

4-(dimethylamino)benzylideneamino)-N(pyrimidine-2-yl)-3-(pyrimidine-yldiazenyl) benzene sulfonamide[B1]:

(3.56 g) of Azo compound, (S1) with (1.49 g) from ( $p-N, N$-dimethyl amino benzaldehyde), the color of yield ( red), (72\%), m.p.(150-152 $\left.{ }^{\circ} \mathrm{C}\right)$, T.L.C. ( Met:tol ) ( 6:4) ( $\left.R_{f} 0.92\right)$.

\section{I.R spectra}

( C=N )str.Imine (w 1640) $\mathrm{cm}^{-1}$, (S .C=N ) Pyrimidine $(1678) \mathrm{cm}^{-1},(\mathrm{C}=\mathrm{C})$ str.Aromatic $(1533-$ 1585) $\mathrm{cm}^{-1},(\mathrm{~N}-\mathrm{H})$ str. Sulfone $(3311) \mathrm{cm}^{-1},(\mathrm{C}=\mathrm{N})$ str. Pyrimidine (w. 1630) $\mathrm{cm}^{-1}$, ,AZO(1440-1494) $\mathrm{cm}^{-1}$, Aliphatic C-H (2931) $\mathrm{cm}^{-1}$, (C-H) str. Pyrimidine ring (3184) $\mathrm{cm}^{-1}$, , (C-H) str. Aromatic (3037-3111) $\mathrm{cm}^{-1}$.

\section{${ }^{1}$ HNMR spectrum}

$(\delta \mathrm{ppm}),(\mathrm{DMSO}-d 6 \mathrm{MHz})(\mathrm{N}-\mathrm{CH} 3) 2$ $(3,058),(\mathrm{H}-\mathrm{C}=\mathrm{N})$ Imine (8.518) (Ar-H) (6.55-7.979),
$(\mathrm{HC}=\mathrm{N})$ pyrimidine (8.002-8.49), $(\mathrm{N}-\mathrm{H})$ Sulfone (11.279), $\mathrm{DMSO}(2.5)$.

\section{${ }^{13} \mathrm{C}$-NMR-spectrum}

( $\delta \mathrm{ppm}),(\mathrm{DMSO}-\mathrm{d6}, \mathrm{MHz}), 31.7,111.55$, $112.58,115.99,125.32,127.91,130.28,132.03$, $153.48,157.69,158.73$.

4-(chlorobenzylideneamino)-N-(pyrimidine-2-yl)3-( pyrimidine-2-yldiazenyl)benzene sulfonamide [B2]:

(3.56 g) of Azo compound, (S1) with (1.405 $\mathrm{g})$ from ( $p$-Chloro benzaldehyde), the color of yield (Brown), $(60 \%)$, m.p. $\left(166-168{ }^{\circ} \mathrm{C}\right)$, T.L.C. (Met: Tol ) ( 6:4) ( Rf, 0.82 ).

\section{I.R spectra}

( $\mathrm{C}=\mathrm{N})$ str. Imine (w. 1640) $\mathrm{cm}^{-1},(\mathrm{C}=\mathrm{C}) \mathrm{str}$. Aromatic (1541-1585) $\mathrm{cm}^{-1},(\mathrm{~N}-\mathrm{H})$ str. Sulfone (3311) $\mathrm{cm}^{-1},(\mathrm{C}=\mathrm{N})$ str. Pyrimidine $(\mathrm{S} .1680) \mathrm{cm}^{-1}$, Azo (1419-1494) $\mathrm{cm}^{-1}$, (C-H) str. Pyrimidine ring(3186) $\mathrm{cm}^{-1},(\mathrm{C}-\mathrm{H})$ str. Aromatic (3037-3111) $\mathrm{cm}^{-1},(\mathrm{C}-\mathrm{Cl})\left(615 \mathrm{~cm}^{-1}\right)$.

\section{${ }^{1} \mathrm{HNMR}$ spectrum}

$(\delta \mathrm{ppm}),(\mathrm{DMSO}-\mathrm{d} 6 \mathrm{MHz}),(\mathrm{H}-\mathrm{C}=\mathrm{N})$ Imine (8.768) (Ar-H) (7.050-7.945), ( $\mathrm{HC}=\mathrm{N})$ pyrimidine (8.035-8.536), ( $\mathrm{N}-\mathrm{H})$ Sulfone (11.079), DMSO (2.5).

\section{${ }^{13} \mathrm{C}$-NMR-spectrum}

$(\delta \mathrm{ppm}),(\mathrm{DMSO}-d 6, \mathrm{MHz}), 116.11$, 118.657, 129.352, 134.276, 143.641, 157.458, 158.809, 169.531.

(4-hydroxy-3-methoxybenzylidineamino)-N(pyrimidine-2-yl)-3-( pyrimidine-2-yldiazenyl) benzene sulfonamide[B3].

(3.56 g) of Azo comp, (S1) with (1.52 g) from Vaniline(p-hydroxy-m-methoxy benzaldehyde), the color of yield ( Orange),(61\%),m.p. $\left(145-146{ }^{\circ} \mathrm{C}\right)$, T.L.C. ( Met:tol ) ( 3:2 ) ( Rf, 0.87 ).

\section{I.R spectra}

( $\mathrm{C}=\mathrm{N})$ str.Imine $(1639) \mathrm{cm}^{-1},(\mathrm{C}=\mathrm{N})$ Pyrimidine (1674) $\mathrm{cm}^{-1},(\mathrm{C}=\mathrm{C})$ str. Aromatic (1577) $\mathrm{cm}^{-1},(\mathrm{~N}-\mathrm{H})$ str. Sulfone $(3377) \mathrm{cm}^{-1},(\mathrm{C}=\mathrm{N}) \mathrm{str}$. Pyrimidine (1639) $\mathrm{cm}^{-1}$, AZO(1438-1492) $\mathrm{cm}^{-1}$, (C-H) str. Pyrimidine ring (3257) $\mathrm{cm}^{-1},(\mathrm{C}-\mathrm{H}) \mathrm{str}$. Aromatic $(3037-3105) \mathrm{cm}^{-1},(\mathrm{OH})\left(3458 \mathrm{~cm}^{-1}\right)$, Aliphatic $\mathrm{C}-\mathrm{H}(2937) \mathrm{cm}^{-1}$. 
${ }^{1}$ HNMR spectrum

$(\delta \mathrm{ppm}),(\mathrm{DMSO}-d 6 \mathrm{MHz}),(\mathrm{H}-\mathrm{C}=\mathrm{N})$ Imine (8.66), $\left(\mathrm{O}-\mathrm{CH}_{3}\right)$ (3.919), (Ar-H) (6. 263-7.247), $(\mathrm{HC}=\mathrm{N})$ pyrimidine $(7.551-8.446),(\mathrm{N}-\mathrm{H})$ Sulfone ( 11.47), (OH) (9.77), DMSO(2.5).

\section{${ }^{13}$ C-NMR-spectrum}

( $\delta$ ppm), (DMSO-d6, MHz), 56.017, $111.047,112.583,115.828,115.992,116.168$, $118.642,125.248,126.603,129.148,129.339$, $130.309,134.306,143.622,148.603,153.515$, $157.486,157.679,158.732,158.810,169.525$.

4-(Bromo benzylideneamino)-N-(pyrimidine-2-yl)3-( pyrimidine-2-yldiazenyl)benzene sulfonamide [B4]:

(3.56 g) of Azo comp, (S1) with (1. 84g) from ( $p$-bromo benzaldehyde), the color of yield (dark goldenrod), (63\%), m.p.( $\left.173-175^{\circ} \mathrm{C}\right)$, T.L.C. ( Met:tol ) ( 1:4 ) ( Rf, 0.55 ).

\section{I.R spectra}

( C=N ) str. Imine (w. 1645) $\mathrm{cm}^{-1},(\mathrm{C}=\mathrm{N})$ str. Pyrimidine $(\mathrm{m} .1672) \mathrm{cm}^{-1},(\mathrm{C}=\mathrm{C})$ str. Aromatic (1579) $\mathrm{cm}^{-1},(\mathrm{~N}-\mathrm{H})$ str. Sulfone $(3271) \mathrm{cm}^{-1}$, Azo compound (1415-1448) $\mathrm{cm}^{-1},(\mathrm{C}-\mathrm{H})$ str. Pyrimidine ring (3186) $\mathrm{cm}^{-1},(\mathrm{C}-\mathrm{H})$ str. Aromatic (3037-3105) $\mathrm{cm}^{-1},(\mathrm{C}-\mathrm{Br}) 798$ $\mathrm{cm}^{-1}$.

\section{${ }^{1} \mathrm{HNMR}$ spectrum}

$(\delta \mathrm{ppm}),(\mathrm{DMSO}-d 6 \mathrm{MHz}),(\mathrm{H}-\mathrm{C}=\mathrm{N})$ Imine (8.840), (Ar-H) (6.889-7.945), ( $\mathrm{HC}=\mathrm{N})$ pyrimidine (8.053 -8. 715), (N-H) Sulfone (11.256), DMSO (2.5).

\section{${ }^{13} \mathrm{C}-\mathrm{NMR}$-spectrum}

( $\delta \mathrm{ppm}),(\mathrm{DMSO}-d 6, \mathrm{MHz}), 113.706$, $115.511,118.562,118.961,123.694,125.248$, $129.192,129.880,130.938,131.741,132.469$, $143.285,143.605,146.942,158.633,160.125$, 169.435.

4-(Nitro benzylideneamino)-N-(pyrimidine-2-yl)-3(pyrimidine-2-yldiazenyl)benzene sulfonamide [B5]:

(3.56 g) of Azo comp,(S1) with (1.51 g) from ( $p$-Nitro benzaldehyde), the color of yield (orange), (76\%), m.p. (202-204 C), T.L.C.( Met:tol ) (2:3 ) ( Rf, 0.64 ).

\section{I.R spectra}

( $\mathrm{C}=\mathrm{N}$ )str.Imine interaction with $(\mathrm{C}=\mathrm{N}$ ) Pyrimidine $(1680) \mathrm{cm}^{-1},(\mathrm{C}=\mathrm{C})$ str.Aromatic (1585)
$\mathrm{cm}^{-1},(\mathrm{~N}-\mathrm{H})$ str. Sulfone (3311) $\mathrm{cm}^{-1}$, Azo compound (1406-1440) $\mathrm{cm}^{-1},(\mathrm{C}-\mathrm{H}) \mathrm{str}$. Pyrimidine ring (3182) $\mathrm{cm}^{-1}$, (C-H) str. Aromatic (3039-3113) $\mathrm{cm}^{-1},\left(\mathrm{NO}_{2}\right)(1336-1535) \mathrm{cm}^{-1}$

\section{${ }^{1}$ HNMR spectrum}

$(\delta \mathrm{ppm}),(\mathrm{DMSO}-\mathrm{d} 6 \mathrm{MHz}),(\mathrm{H}-\mathrm{C}=\mathrm{N})$ Imine (S 8.893), (Ar-H) (m 7.068-7.981), (HC=N) pyrimidine (m 8.339-8.643, 9.233), (N-H) Sulfone (11.363), DMSO(2.5).

\section{${ }^{13}$ C-NMR-spectrum}

( $\delta$ ppm), (DMSO- $d 6, \mathrm{MHz}), 116.421$, 118.348, 122.842, 124.554, 129.476, 131.188, 132.044, 133.756, 143.815, 157.939, 159.651, 169.710

\section{Preparation of $\beta$-Lactam derivatives[L1-L5] from} Schiff bases ${ }^{17}$ :

To a mixture of Schiff base $(0.01 \mathrm{~mol})$ in dioxane $(25 \mathrm{ml})$ and triethylamine $(3.49 \mathrm{ml}$, $0.025 \mathrm{~mol})$, was added chloro acetyl chloride $(1.99 \mathrm{ml}, 0.025 \mathrm{~mol})$ drop-wise at $(5-10){ }^{\circ} \mathrm{C}$. The reaction mixture was stirred for $(6 \mathrm{~h})$ and kept at room temperature for two days then poured into crushed ice. The solvent was evaporated and the yalid recrystallized from ethanol. All the reactions was monitored by Thin Layer Chromatography.

4-(3-chloro-2-(4-(dimethylamino)phenyl)-4oxoazitidine-1-yl)-N-(pyrimidine-2-yl)-3(pyrimidine-2-yldiazenyl) benzene sulfonamide[L1]:

It was prepared from shiff base [B1](4.87 g, 0.01 mole), $\mathrm{Et}_{3} \mathrm{~N}(3.49 \mathrm{ml}, 0.025$ mole) and $\mathrm{ClCH}_{2} \mathrm{COCl}$ (1.99 ml, $0.025 \mathrm{~mole}$ ), the color (Brown), $63 \%$, m.p. (Gummy), T.L.C. (Met:tol) ( 1:4 ) ( Rf, 0.41 ).

\section{I.R spectra}

$(\mathrm{C}=\mathrm{O})$ str. (S.1743) $\mathrm{cm}^{-1},(\mathrm{C}=\mathrm{N})$ Pyrimidine (1662), (C=C) str.Aromatic (1595) $\mathrm{cm}^{-1},(\mathrm{~N}-\mathrm{H})$ str. Sulfone (3404) $\mathrm{cm}^{-1}$,Azo compound (1436-1463) cm ${ }^{1},(\mathrm{C}-\mathrm{H})$ str. Aliphatic(2854-2929) $\mathrm{cm}^{-1},(\mathrm{C}-\mathrm{Cl}) 572 \mathrm{~cm}^{-1}$.

\section{${ }^{1}$ HNMR spectrum}

( $\delta$ ppm), (DMSO-d6 MHz), $\left(\mathrm{N}-\mathrm{CH}_{3}\right)$ (S2.827), (N-CH) ( d.5.440), (CO-CH-Cl)(d.5.85), $(\mathrm{Ar}-\mathrm{H})(7.516-8.142),(\mathrm{HC}=\mathrm{N})$ pyrimidine $(8.607-$ 9.325), (N-H) Sulfone (11.184), DMSO(2.5). 
${ }^{13} \mathrm{C}-\mathrm{NMR}$-spectrum

( $\delta$ ppm), (DMSO-d6 ,MHz),46, 62, 64, 116.64, 118.78, 121.77, 125.84, 127.12, 129.05, 133.33,135.25,139.53,141.67,157.08,159.22, $169.71,172$.

4-(3-chloro-2-(4-(chloro phenyl)-4-oxoazitidine-1yl)-N-(pyrimidine-2-yl)-3-(pyrimidine-2yldiazenyl)benzene sulfonamide[L2]:

Was prepared from shiff base [B2](4.785 g, 0.01 mole), $\mathrm{Et}_{3} \mathrm{~N}(3.49 \mathrm{ml}, 0.025$ mole) and $\mathrm{ClCH}_{2} \mathrm{COCl}(1.99 \mathrm{ml}, 0.025$ mole) , the color (pale yallow), 50\%, m.p. (Oily), T.L.C. ( Met:tol) ( $1: 4$ ) ( Rf, 0.60 ).

\section{I.R spectra}

( $\mathrm{C}=\mathrm{O})$ str. $\quad(\mathrm{S} .1741) \mathrm{cm}^{-1}, \quad(\mathrm{C}=\mathrm{C})$ str.Aromatic (1595) $\mathrm{cm}^{-1},(\mathrm{~N}-\mathrm{H})$ str. Sulfone (3415) $\mathrm{cm}^{-1}$, Azo compound (1462) $\mathrm{cm}^{-1},(\mathrm{C}-\mathrm{H})$ str. Aliphatic(2937-2981) $\mathrm{cm}^{-1}$, (C-Cl) (w.582 $\mathrm{cm}^{-1}$.

\section{${ }^{1} \mathrm{HNMR}$ spectrum}

( $\delta \mathrm{ppm}),(\mathrm{DMSO}-\mathrm{d} 6 \mathrm{MHz})),(\mathrm{N}-\mathrm{CH})$ (d.3.740), (CO-CH-Cl) (d.4.062), (Ar-H) (7.7138.607), $(\mathrm{HC}=\mathrm{N})$ pyrimidine (8. 786-9.341), $(\mathrm{N}-\mathrm{H})$ Sulfone (10.934).

\section{${ }^{13} \mathrm{C}-\mathrm{NMR}$-spectrum}

( $\delta \mathrm{ppm}),(\mathrm{DMSO}-\mathrm{d6}, \mathrm{MHz}), 58.211,68.269$, $110.429,114.281,117.277,121.558,124.982$, $129.690,130.974,134.826,139.320,145.313$, $149.379,151.519,161.149,165.002,172.706$, 182.122

4-(3-chloro-2-(4-(hydroxyl-3-methoxy phenyl)-4oxoazitidine-1-yl) -N-(pyrimidine-2-yl)-3(pyrimidine-2-yldiazenyl)benzene sulfonamide [L3]:

It was prepared from shiff base [B3] $(4.9 \mathrm{~g}$, 0.01 mole), $\mathrm{Et}_{3} \mathrm{~N}(3.49 \mathrm{ml}, 0.025 \mathrm{~mole})$ and $\mathrm{ClCH}_{2} \mathrm{COCl}(1.99 \mathrm{ml}, 0.025$ mole), the color (pal Brown), 57\%, m.p. (Oily), T.L.C. ( MET:TOL ) ( $3: 2)\left(R_{f}, 0.45\right)$.

\section{I.R spectra}

( $\mathrm{C}=\mathrm{O})$ str. $(1734) \mathrm{cm}^{-1},(\mathrm{C}=\mathrm{C})$ str.Aromatic (1587) $\mathrm{cm}^{-1},(\mathrm{~N}-\mathrm{H})$ str. Sulfone $(3317) \mathrm{cm}^{-1}$, Azo compound (1438-1475) $\mathrm{cm}^{-1},(\mathrm{C}-\mathrm{H})$ str. Aliphatic (2937-2976) $\mathrm{cm}^{-1}$, (C-Cl) $572 \mathrm{~cm}^{-1}$.

\section{${ }^{1}$ HNMR spectrum}

( $\delta \mathrm{ppm}),(\mathrm{DMSO}-d 6,400 \mathrm{MHz})),(\mathrm{N}-\mathrm{CH}-\mathrm{C})$ (d2.379), (CO-CH-Cl) ( d.2.734-2.776), $\left(3 \mathrm{H}, \mathrm{OCH}_{3}\right)$ (s,3.282), (Ar-H) (6.366-6.649), $(\mathrm{HC}=\mathrm{N})$ pyrimidine (6853-7.545), (OH) (s 9.153), (N-H) Sulfone (10.761), DMSO(2.5).

\section{${ }^{13} \mathrm{C}$-NMR-spectrum}

( $\delta$ ppm), (DMSO- $d 6, \mathrm{MHz}), 27.15,64.42$, $83.79,113.13,124.05,131,136,152.81,153$, $158.51,170.54$.

4-(2-(4-Bromo phenyl)-3-chloro-4-oxoazitidine-1yl)-N-(pyrimidine-2-yl)-3-(pyrimidine-2yldiazenyl)benzene sulfonamide[L4]:

It was prepared from schiff base [B4] (5.229 g, 0.01 mole), $\mathrm{Et}_{3} \mathrm{~N}(3.49 \mathrm{ml}, 0.025$ mole $)$ and $\mathrm{ClCH}_{2} \mathrm{COCl}$ (1.99 ml, 0.025 mole), the color yallow, m.p. (55-57) ${ }^{\circ} \mathrm{C}$, T.L.C. ( Met: Tol ) (3:2) ( $\left.\mathrm{R}_{f} 0.86\right)$.

\section{I.R spectra}

$(\mathrm{C}=\mathrm{O})$ str. (S.1743) $\mathrm{cm}^{-1},(\mathrm{C}=\mathrm{N})$ Pyrimidine (1627) $\mathrm{cm}^{-1},(\mathrm{C}=\mathrm{C})$ str.Aromatic $(1598) \mathrm{cm}^{-1},(\mathrm{~N}-\mathrm{H})$ str. Sulfone (3417) $\mathrm{cm}^{-1}$,Azo compound(1463) $\mathrm{cm}^{-1}$, (C-H) str. Aliphatic (2922-2983) $\mathrm{cm}^{-1}$.

\section{'HNMR spectrum}

( $\delta \mathrm{ppm}),(\mathrm{DMSO}-\mathrm{d} 6 \mathrm{MHz})),(\mathrm{N}-\mathrm{CH}-\mathrm{C})$ (d 3.543), (CO-CH-Cl) ( d.4.992), (Ar-H) (m 7.1227.677), $(\mathrm{HC}=\mathrm{N})$ pyrimidine (m 7.820-8.643), $(\mathrm{N}-\mathrm{H})$ Sulfone (s 11.166) ,DMSO(2.5).

4-(3-chloro-2-(4-(nitro phenyl)-4-oxoazitidine-1yl)-N-(pyrimidine-2-yl)-3-(pyrimidine-2yldiazenyl)benzene sulfonamide[L5]:

It was prepared from shiff base [B5] (4.89g, 0.01 mole $), \mathrm{Et}_{3} \mathrm{~N}(3.49 \mathrm{ml}, 0.025$ mole $)$ and $\mathrm{ClCH}_{2} \mathrm{COCl}$ (1.99 ml, 0.025 mole), the color (Green yellow), m.p. (123-125) C, T.L.C. (Met:tol) ( 1:4 ) ( Rf, 0.45).

\section{I.R spectra}

$(\mathrm{C}=\mathrm{O})$ str. (1735) $\mathrm{cm}^{-1},(\mathrm{C}=\mathrm{N})$ Pyrimidine (1678) $\mathrm{cm}^{-1},(\mathrm{C}=\mathrm{C})$ str.Aromatic $(1585) \mathrm{cm}^{-1},(\mathrm{~N}-\mathrm{H})$ str. Sulfone (3392) $\mathrm{cm}^{-1}$, Azo compound(1442-1465) $\mathrm{cm}^{-1},(\mathrm{C}-\mathrm{H})$ str. Aliphatic(2850-2920) $\mathrm{cm}^{-1}$.

\section{'HNMR spectrum}

( $\delta \mathrm{ppm}),(\mathrm{DMSO}-\mathrm{d} 6 \mathrm{MHz})),(\mathrm{N}-\mathrm{CH}-\mathrm{C})$ (d. 5. 350), (CO-CH-Cl) ( d.5.798), (Ar-H) (m 6.6757.820), $(\mathrm{HC}=\mathrm{N})$ pyrimidine $(\mathrm{m} 8.178-9.162),(\mathrm{N}-\mathrm{H})$ Sulfone(s 11.143), DMSO(2.5). 
${ }^{13} \mathrm{C}-\mathrm{NMR}$-spectrum

( $\delta$ ppm), (DMSO-d6, MHz), 112.569, 115.351, 118.775, 125.624, 129.262,134.612, 137.822, 143.601,147.025, 150.449, 158.795, 166.072, 169.924,187.687.

\section{RESULTS AND DICUSSION}

Sulphaziazine was chosen as a starting material for the synthesis of new Azo compound. (S1) through a diazo-coupling reactionwith (2-amino pyrimidine) scheme ${ }^{1}$. the appearance of stretching band of primary aromatic amine $(\mathrm{NH} 2)$ at (3427$3358) \mathrm{cm}^{-1}$ and Azo group $(\mathrm{N}=\mathrm{N})$ at $(1440-1409)$ $\mathrm{cm}^{-1}$, and showed ${ }^{1} \mathrm{HNMR}$ spectrum of the compound characteristic signals: $\left(6.015,2 \mathrm{H},-\mathrm{NH}^{2}\right)$, (11.294, $1 \mathrm{H}, \mathrm{NH}$-Salfone) are attributed to the formation of Azo compound.

The condensation reaction of Azo compound. with different aromatic aldehydes in the presence of glacial Acetic acid as solvent Scheme 2 gave the corresponding Schiff bases derivatives (B1-B5). The disappearance of the absorption bands at (34273358) $\mathrm{cm}^{-1}\left(-\mathrm{NH}_{2}\right)$ and appearance absorption bands of $(\mathrm{C}=\mathrm{N})$ Imine at (1639-1680 interaction with $\mathrm{C}=\mathrm{N}$ pyrimidine rings) $\mathrm{cm}^{-1}$ were confirm the structures of the compounds (B1-B5) ${ }^{1} \mathrm{HNMR}$ spectrum of these Schiff bases showed the signal of imine proton at about (8.5-8.8 ppm).

Schiff bases (B1-B5) that reacted with chloro acetyl chloride in the presence of tri ethyl amine in dry dioxane by a [2+2] cyclo addition reaction Scheme ${ }^{3}$ to give corresponding $\beta$-lactam derivatives (L1-L5) ${ }^{18}$. appearance of the absorption bands of carbonyl lactam ring at (1734-1743) $\mathrm{cm}^{-1}$, these stretching frequencies in monocyclic lactams indicates that the carbonyl group in $\beta$-lactam behaves like" ester" group ${ }^{19}$. The spectra ${ }^{13} \mathrm{C}-\mathrm{NMR}$ of carbonyl Lactam

Showed the typical resonance at $\delta$ (169-187) ppm. the values outside this range are possible if strong electron donating or electron withdrawing groups are present on the adjacent carbon atoms. example for this, the spectrum ${ }^{13} \mathrm{CNMR}$ of $(\mathrm{L} 1, \mathrm{~L} 3)$ showed the carbonyl carbon signal at $(169.71,170.54)$ respectively. where as the carbonyl group in (L5) resonated at $(182,187.687) \mathrm{ppm}^{17}$.
The mecanisim of mono- $\beta$-lactam formation via [2+2] cyclo addition showen in the Scheme $(4)^{21}$.

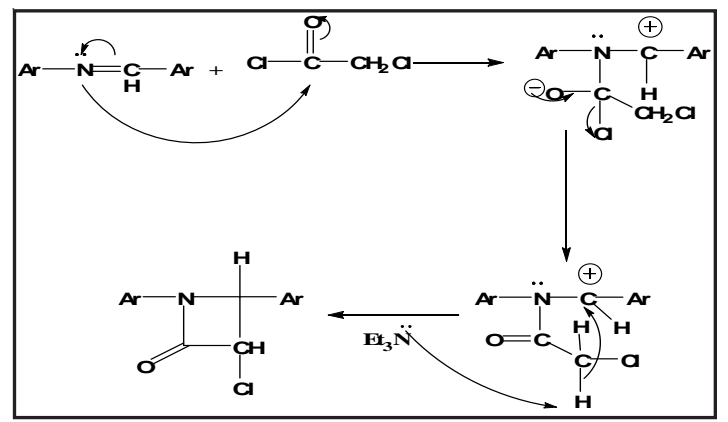

Scheme. 4: mecanisim of [2+2] cyclo addition

Biological part

2-Azetidinone Derivative as anticancer

\section{MATERIALS AND METHODS}

\section{Methyl thiazolyl tetrazolium (MTT) Solution}

Methyl thiazolyl tetrazolium $(0.2 \mathrm{~g})$ (Bio-world, USA) was dissolved in $100 \mathrm{ml}$ of PBS in order to prepare a $2 \mathrm{mg} / \mathrm{ml}$ concentration of the dye. The solution was filtered through $0.2 \mu \mathrm{m}$ syringe filter to remove any blue formazan product, and then stored in sterile, dark, screw-capped bottles at $4{ }^{\circ} \mathrm{C}$. The solution was used within no longer than 2 weeks of preparation.

\section{Cytotoxicity assay ${ }^{22,23:}$}

MTT cell viability assay was conducted on 96-well plates (Santacruz Biotechnology, USA), MCF7 Human Breast cancer cells were seeded at 10000 cells/well, $200 \mu$ l of cells in growth medium were added to each well of a sterile 96-well microtitration plate. The plates were sealed with a self-adhesive film, lid placed on and incubated at $37^{\circ} \mathrm{C}$.

After $24 \mathrm{~h}$ or confluent monolayer is achieved, when the cells were in exponential growth, the medium was removed and serial dilutions of the extract were added to the wells at 2-fold serial dilutions (dilution the stock1/10 ml). triplicates were used for each. Control cells treated with Serum Free Media only. Afterwards, the plates were re-incubated at $37^{\circ} \mathrm{C}$ for 72 hours.

Cell viability was measured after $72 \mathrm{~h}$ of exposure by removing the medium, adding $28 \mu \mathrm{l}$ of $2 \mathrm{mg} / \mathrm{ml}$ solution of MTT (Bio-World, USA) and incubating for $1.5 \mathrm{~h}$ at $37^{\circ} \mathrm{C}$. After removing the MTT 
solution, the crystals remaining in the wells were solubilised by the addition of $130 \mu$ of DMSO (Dimethyl Sulphoxide) (Santacruz Biotechnology, USA) followed by $37^{\circ} \mathrm{C}$ incubation for $15 \mathrm{~min}$. with shaking.

The absorbency was determined on a microplate reader (Biochrom, UK) at $584 \mathrm{~nm}$ (test wavelength); the assay was performed in 5 replicates.

\section{Endpoint parameters that are calculated for each individual cell line included:}

1- Percentage of cell growth or percentage of cell proliferation $(\mathrm{PR})=$ mean of treatment / mean of control.

2- The lowest concentration that kills $50 \%$ of cells (LC50).

\section{Cell culture for Normal embrynoic cells}

The rat embryo fibroblast (REF) cell line were cultured in a RPMI-1640 medium with $10 \%$ fetal bovine serum (FBS), 100 units/mL penicillin, and $100 \mu \mathrm{g} / \mathrm{mL}$ streptomycin and then incubated at $37{ }^{\circ} \mathrm{C}$. All the cell lines were supplied by thelraqi Biotechnology Company, Cell culture lab, Baghdad,
Iraq. These cells are regularly assessed for standard growth characteristics, and they are regularly authenticated.

The anticancer activity of the synthesized derivative(L1) in vitro was determined against an human breast MCF-7 cancer cell line using MTT cell viability assay. In general the result of the synthesized compound showed better anticancer potential $($ IC50 $=2.900 \mu \mathrm{M})$ after 72 hours.

In Fig. (33) shows that the toxicity effect for [ 4-(3-chloro-2-(4-(di methyl amino)phenyl) -4-oxoazitidine-1-yl)-N-(pyrimidine-2-yl)-3-( pyrimidine-2-yldiazenyl)benzene sulfonamide][L1], which increase with increase the concentration.

In Fig. (34) shows the half lethal concentration which equal (LC50 $2.9 \mu \mathrm{M}$ ) which kill the half number of cell and it will be absolutely lethal with increase concentration. but (LC50) for normal embrynoic cells equal $(9.59 \mu \mathrm{M})$ as shown in Fig. (36) This means that the derivative is likely to be a killer of the cancer cell without affecting the normal cells when using its lethal concentration for half cells.

Table. 1: Cell viability Values

\begin{tabular}{|c|c|c|c|c|c|c|c|c|}
\hline Conc. (uM) & 0.07 & 0.155 & 0.31 & 0.625 & 1.25 & 2.5 & 5 & 10 \\
\hline Cell viability & 78.1 & 74.33 & 68.755 & 73.2 & 66.34 & 61.28 & 50.08 & 50 \\
\hline
\end{tabular}

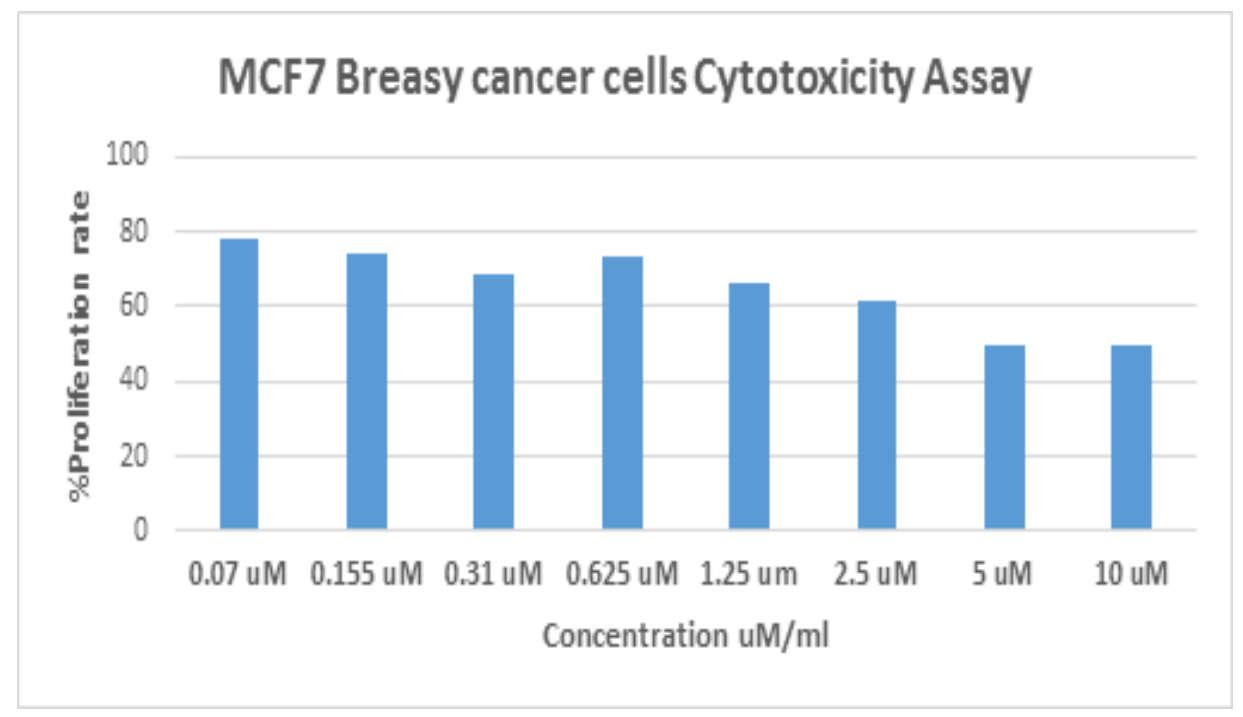

Fig. 1. Cytotoxicity Assay of B-Lactam derivative(L1) on MCF7 Breasy cancer 


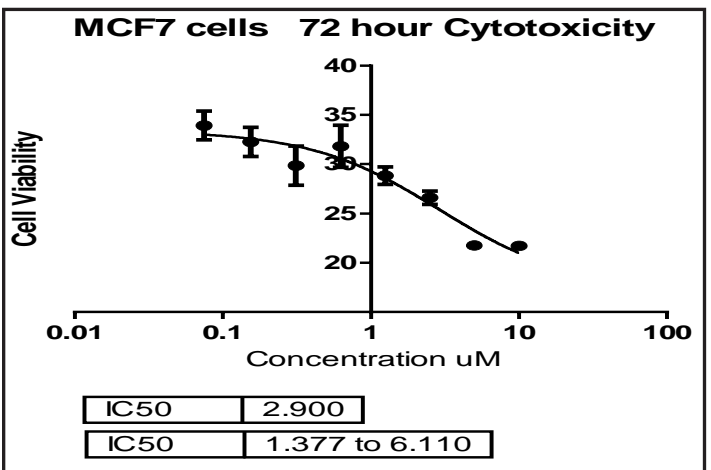

Fig. 2. (IC50) of $\beta$-Lactam derivative(L1) forMCF7 Breasy cancer

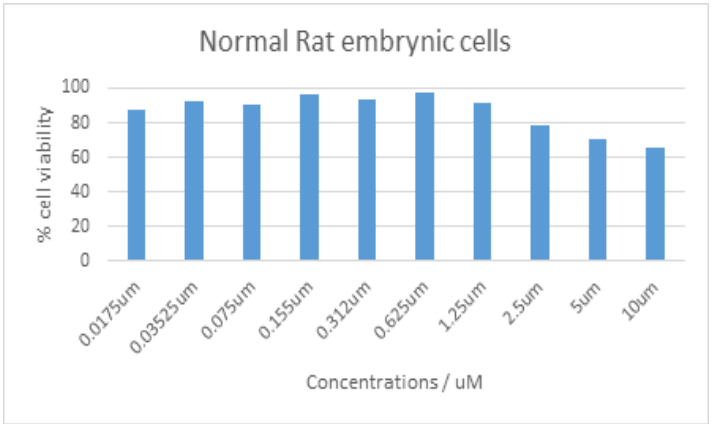

Fig. 3. Cytotoxicity Assay of B-Lactam derivative(L1) on normal rat embrynoic

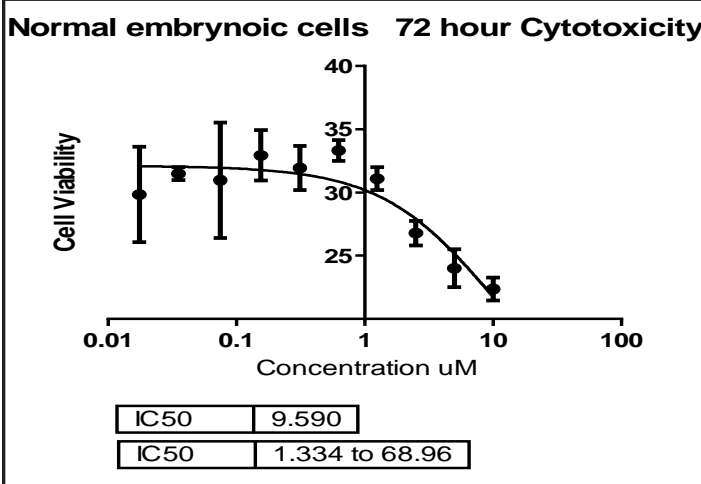

Fig. 4. (IC50) of B-Lactam derivative(L1) for normal rat embrynoic cells

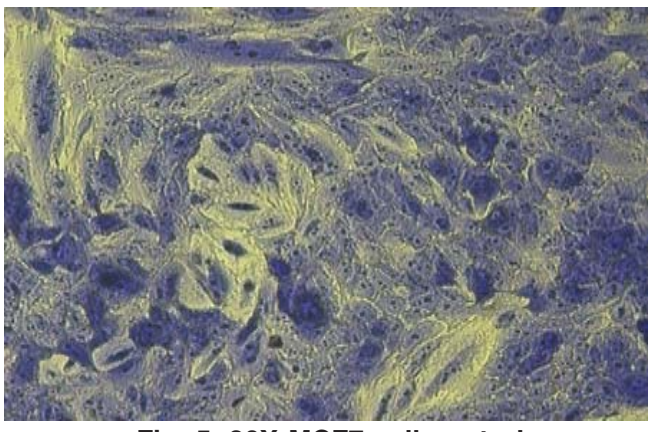

Fig. 5. 20X MCF7 cell control

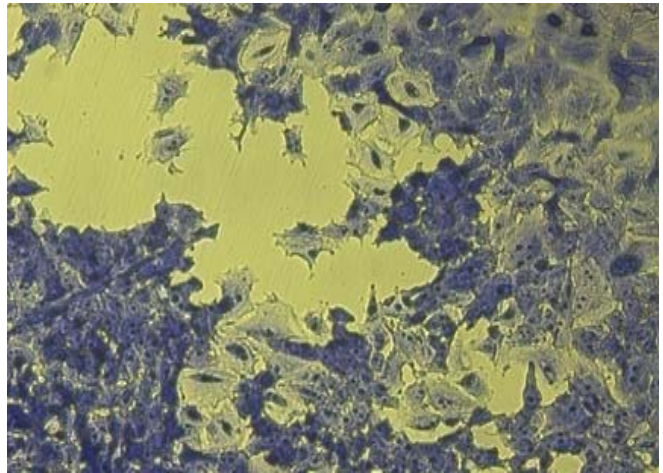

Fig. 6. 20X MCF7 cell treated with best concentration of B-Lactam derivative(L1).

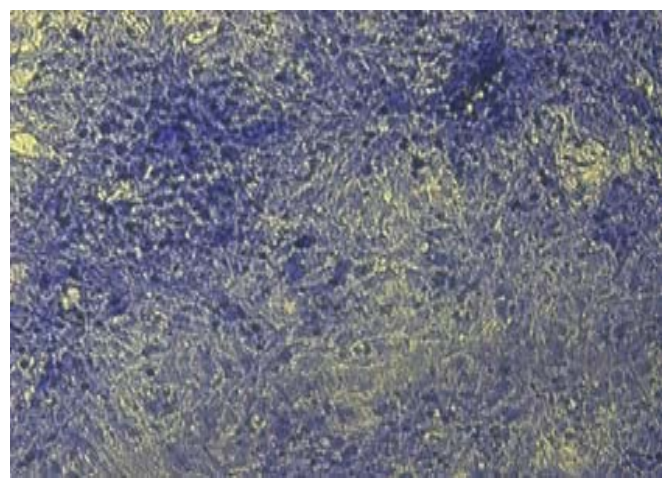

Fig. 7. 20X REF cell Control

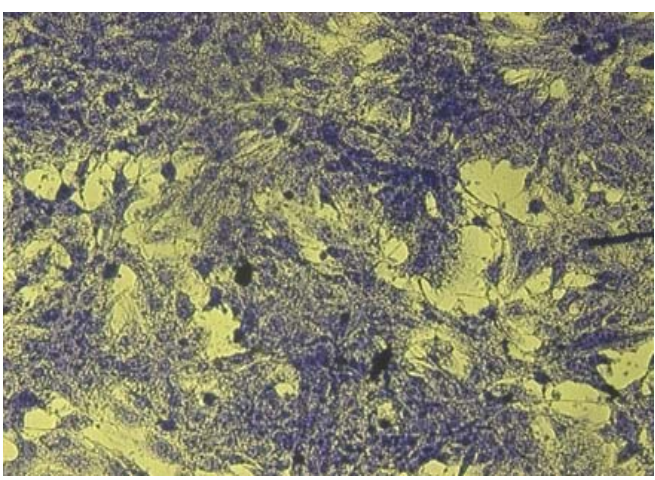

Fig. 8. 20X REF cell treated with best conc. of $\beta$-Lactam derivative(L1) REF (Rat Embryonic Fibroblasts)

\section{CONCLUSION}

In this study we are reported synthesis of many $\beta$-Lactam derivatives via, Staudinger Reaction[2+2]cyclo addition. The work included preparation of Azo-Schiff base compounds from 
sulphadiazine as the first step then cyclization process for these compounds by using chloro acetyl chloride in the basic medium and low temp. $(0-10){ }^{\circ} \mathrm{C}$ These derivatives were found to be stable at room temperature and some of them possess strength oily or sticky. These derivatives confirmed from spectral data analysis; FTIR, ${ }^{1} \mathrm{HNMR}$ and ${ }^{13}$ CNMR.

\section{ACKNOWLEDGMENT}

The authors are thankful to Prof. Dr. Ahlam Kadhum Naeem Al-yasseen, College of education for girls-biology, Kufa University and Iraqi Center for Cancer and Medical Genetic Research, Baghdad, Iraq. Also we really appreciate the help of Mr.Abbas almulla.

\section{REFERENCES}

1. Salim Mohammed, A. ; Zebary, H. Y. S.;" Spectrophotometric Determination of Sulfadiazine via Diazotization and Coupling Reaction - Application to Pharmaceutical Preparations", Raf. J. Sci, 2013, 24(6) 61-73.

2. Who Model List of Essential Medicines; " World Health Organization. October 2013, Retrieved 22 April 2014.

3. Ugras, H.I.; Basaran,I.; Kilic ,T. ; Cakir, U. J. Heterocyclic Chem., 2006, 43 : 1679.

4. Chatterjee, S. ; Bhattacharyya,S.; "Schiff Bases As a Source of Potent Molecules with Anti-cancer Potential: A Short Review",Asian Journal of Biochemical and Pharmaceutical Research, 2015, 5(4) 86-97.

5. Al-Assadi, K, M.J.;" Synthesis and Characterization of $\mathrm{Ni}^{2+}$ and $\mathrm{Cu}^{2+}$ Schiff-base Complexes and Their Study for Electrical Properties", Journal of Basrah Researches ((Sciences)), 2011, 37, (3).

6. Sonnekar, VS. ; Jadhav, W.N. ; Dake, SA. ; Pawar, RP. .;" Synthesis and Antimicrobial and antifungal activities of novel Bis-imine Derivatives", Research Journal of Pharmaceutical, Biological and Chemical Sciences(RJPBCS), 2013, 4 (2) 1411-1418.

7. Mohamed ,S. S.; Al-bashier, S. M.; Shalfoh, E. S. ; Fhid ,O.;" Microwave Assisted one-pot Synthesis and Screening of some schiff's bases of Sulfanilamide", Journal of Chemical and Pharmaceutical Research, 2012, 4(5), 2512-2516.

8. Gharamaleki, J. A.; Akbari, F.; Karbalaei, A.; Ghiassi , K. B. ; Olmstead, M. M. ;"Synthesis, Characterization and Crystal Structure of a New Schiff Base Ligand from a Bis(Thiazoline) Template and Hydrolytic Cleavage of the Imine Bond Induced by a Co(II) Cation", Open Journal of Inorganic
Chemistry, 2016, 6, 76-88.

9. van der, F. S. ; Koten ,G. v. ;" Syntheses of 3Amino-2-azetidinones: A Literature Survey", Tetrahidron , 1991, 41(36) 7503-7524 .

10. Gilchrist, T.," Heterocyclic Chemistry". Harlow: Longman Scientific (1987).

11. Ehmann, D. E.; Avibactam is a covalent, reversible, non- $\beta$-lactam $\beta$-lactamase inhibitor". PNAS. , 2012, 109(29), 11663-11668.

12. Tidwell , Thomas T.; "Hugo (Ugo) Schiff, Schiff Bases, and a Century of â-Lactam Synthesis". Angewandte Chemie International Edition, 2008, 47 (6), 1016-20.

13. Staudinger, H.; "Zur Kenntniss der Ketene. Diphenylketen". Justus Liebigs Ann. Chem., 1907, 356 (1-2), 51-123.

14. Nangia, A.; Biradha, K.; Desiraju, G.R.; "Correlation of biological activity in â-lactam antibiotics with Woodward and Cohen structural parameters: A Cambridge database study". J Chem Soc, Perkin Trans., 1996, 2(5), 943-53.

15. Naser, A. W. ; Majeed ,A. M.; "Synthesis of some new sulfonamide derivatives based on 1,3,4-oxadiazole"., Journal of Chemical and Pharmaceutical Research, 2015, 7(3), 300-306.

16. Anusha, K. ; Pradeep Kumar, Y.; Vara Prasad, M. ; raju V. B. m. ; Gopinath ,C.;"A review on 2azetedinones", Journal of Global Trends in Pharmaceutical Sciences( JGTPS), 2015, 6(1), (12388 - 2402).

17. Magtoof, M . S. ; Hassan, Z. S.;"Synthesis and Characterization Of Some 3-Phenylthio/3Phenoxyazetidine-2- One: Application of Two Dimensional NMR HMQC 1H-13C, Cosy $1 \mathrm{H}-1 \mathrm{H}$ And Mass Spectroscopy" National Journal of Chemistry(NJC), 2011, 41, 90- 105

18. Parmar ,K. ; Modi, V. ; Prajapati, S. ; Patel, R., ;A Facile And Expeditious Approach For The 
Synthesis Of 2-Azetidinone Derivatives With Microbial Activity".,Asian Journal of Biochemical and Pharmaceutical Research, 2011, 2 (1),2231-2560 .

19. yaqoob, R. H. ; Fahad, T.A. ;" Preparation, characterization and antimicrobial activity of new azo complexes containing paracetamol moiety", $J$ of world of pharmaceutical Research, 2015, 5, 01,35-43.

20. Abbass. A. F. ; Zimam ,E. H.; "Synthesis , characterization and study biological activity of some new pyrimidine and 1, 2, 3, 4tetrazole derivatives based on sulfadiazine",
International Journal of ChemTech Research 2016, 9, (11), 206-217.

21. Mohammed ,H. ; Zimam, E. H. ,Journal of Kufa for Chemical Science, 2012, 6, 130-141.

22. Freshney,R.I., of animal cells: $A$ manual for basic technique, $5^{\text {th }}$ ed., John Wily \& sons, lanc. publication, New York, (2005).

23. Al-Shammari, A. M.; Salman, M. I.; Saihoodm Y. D.; Yaseen ,N. Y.; Raed K.;Shaker, H. K.; Ahmed, A.; Khalid, A. ; Duiach, A.," In Vitro Synergistic Enhancement of Newcastle Disease Virus to 5-Fluorouracil Cytotoxicity against Tumor Cells"Biomedicines, 2016, 4, 3. 